\title{
Spatial Analysis of Chinese Residents' Grain Intake
}

\author{
Fuxin JIANG ${ }^{1,2, a}$, Puxiu YUE ${ }^{3, b, *}$, Xiaoning CUI ${ }^{1,2, c}$ \\ ${ }^{1}$ School of Economics and Management, University of Chinese Academy of Sciences, Beijing 100190, China \\ ${ }^{2}$ Academy of Mathematics and Systems Science, Chinese Academy of Sciences, Beijing 100190, China \\ ${ }^{3}$ School of Information, Beijing Wuzi University, Beijing 101149, China

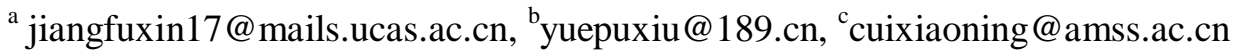

\begin{abstract}
In this paper, spatial exploratory analysis and spatial hierarchical clustering method are used to analyze the similarity and difference of grain intake in China's provinces and cities. The results of global Moran index show that the grain intake of residents of provinces and cities in China is positively correlated. The results of the local Lisa index show that the flour intake of residents in northern China presents a High-High aggregation pattern, the rice intake of residents in southern China presents a High-High aggregation pattern. Through the spatial hierarchical cluster analysis, the main grain intake in the northern provinces of China is flour, and the main grain intake in the southern provinces is rice. Through the analysis of the spatial correlation of grain intake of residents in each province and city, it is helpful for the transportation of grain in neighboring provinces and cities when they are short of production.
\end{abstract}

Keywords: spatial exploratory analysis, spatial hierarchical clustering, Moran index, Lisa index

\section{Introduction}

The food issue has always been a major and important strategic issue concerning China's national economic development, social stability and national independence.Safeguarding China's food security is of great significance to realizing the goal of building a well-off society in an all-round way, building a socialist harmonious society, and promoting the building of a new socialist countryside. In the 2016 Global Hunger Index, China scored 7.7 points, down 3.8 points from 2008, indicating that China's food security situation has improved. At the same time, the periodic surplus characteristics of corn and rice are obvious, the supply of high-quality wheat varieties is insufficient, and the grain problem in China has changed from insufficient total amount to structural contradiction. The regional and variety contradiction of supply and demand will be the manifestation of China's grain in the future $^{[1]}$. Influenced by natural and economic factors, China's grain growth center has shifted from the south to the north and from the east to the middle since the $1970 \mathrm{~s}-1980 \mathrm{~s}^{[2-3]}$. The traditional pattern of "south to north grain transfer" in China has been replaced by the pattern of "north to south grain transfer", and the pattern of grain production has changed ${ }^{[4-5]}$. At the same time, each region has gradually formed its own social and economic structure and agricultural development orientation, which is affected by population movement, processing industry, animal husbandry and other industrial development and urbanization development, grain consumption has formed significant regional differences, thus forming the unbalanced regional development of grain production and demand $^{[6-8]}$. In the study of regional distribution pattern of grain, spatial autocorrelation ${ }^{[9]}$, geographic information system $(\mathrm{GIS})^{[10]}$, MI index ${ }^{[11]}$, ESDA, barycenter migration, $\mathrm{GWR}^{[12]}$ and other methods are mostly used to measure the spatial pattern of grain.

\section{Survey data description}

Based on the network of students studying at the University of Chinese Academy of Sciences, this paper conducts a nationwide sample survey of dietary food consumption among residents. Compared

Corresponding author: Puxiu Yue. 
with the traditional national sample survey, the sampling based on the network of undergraduate college students can ensure the integrity of the population structure. Moreover, the direct sample students as the researcher to promote the entire research process, so as to maximize the enthusiasm of the researchers and the target, to ensure the quality of the survey data.

Based on the regional distribution of the national population, some students were selected from more than 40,000 students of the Chinese Academy of Sciences, and a national survey was conducted for the family or relatives of the student. The stratified sampling takes the province as the reginal layer, and at the same time with the urban-rural intersection, except for Hong Kong, Macao, and Taiwan, there are 31 regional layers, and intersect with urban and rural areas, a total of 62 layers. According to the regional distribution, the extracted students act as network nodes (direct samples), and the network fulcrums (indirect samples) are derived from the nodes, and each node corresponds to 10 branches. Using direct samples to select indirect samples, one student node can get 5 individuals surveyed by men and women, and a total of 10 individuals surveyed. Using short -term continuous follow-up surveys and daily records, the current research year is 2017, 2018 and the research time is 3 days, divided into 2 working days (Monday to Friday), 1 day off (Saturday or Sunday). The survey covers 31 provinces and cities in China. Among them, Shandong, Henan, and Hebei have the largest number of samples, accounting for $11.1 \%, 9.6 \%$, and $7.4 \%$ of the total number of people recovered. Overall, the results of this survey are representative from the distribution of provinces. The survey included the daily intake of food, vegetables and fruits, livestock and poultry, eggs and aquatic products, milk, alcohol and beverages, as well as the dining place, the number of people dining together, and the proportion of meals outside. The characteristics of dietary consumption behavior such as ordering take-away ratio. Shanxi, Inner Mongolia sand Heilongjiang have the largest daily grain intake per capita, Guangdong, Fujian and Guangxi have the least daily grain intake per capita.

\section{Spatial Exploratory Analysis of China's Grain Intake}

\subsection{Research methods}

Exploratory spatial data analysis technology is a kind of exploration method that uses data to represent itself. It closely combines statistical analysis of data and map location to highlight spatial relationships, visualize the spatial distribution of research objects, and reflect the space of things. Agglomeration mode and its difference from surrounding things. The ESDA method includes two categories: global autocorrelation and local autocorrelation analysis.

\subsubsection{Global autocorrelation}

The global autocorrelation analysis mainly describes the agglomeration of spatial distribution of regional economic activities from the overall regional space. Explore the overall spatial associations and differences of an attribute in a region. The Moran index is a commonly used measure of autocorrelation, and its formula is as follows:

$$
\text { Moran's } I=\frac{\sum_{i=1}^{n} \sum_{j=1}^{n} W_{i j}\left(Y_{i}-\bar{Y}\right)\left(Y_{j}-\bar{Y}\right)}{S^{2} \sum_{i=1}^{n} \sum_{j=1}^{n} W_{i j}}
$$

Where $S^{2}=\frac{1}{n} \sum_{i=1}^{n}\left(Y_{i}-\bar{Y}\right)^{2} \quad \bar{Y}=\frac{1}{n} \sum_{i=1}^{n} Y_{i} . \mathrm{n}$ is the total number of study areas, $W_{i j}$ is a binary

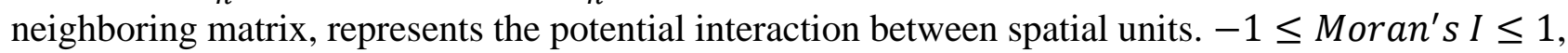
I $>0$ represents spatial self-positive correlation, spatial entities are aggregated distributed; $\mathrm{I}<0$ represents spatial self-negative correlation, spatial entities are discretely distributed; I=0 represents that the spatial entities are randomly distributed.

\subsubsection{Local autocorrelation}

Local autocorrelation analysis can explore the variability of local space, generally measured by Moran scatter plots and local indicators (Lisa). The horizontal axis of the Moran scatter plot is the observed vector $(Z)$ of a variable at different positions, and the vertical axis represents the spatial lag (WZ) of the vector. The scatter plot is divided into 4 quadrants, the first quadrant indicates that the area itself and surrounding areas have higher levels and less spatial differences(High-High); the second quadrant indicates that the area itself is at a lower level and the surrounding area is higher(Low-High); the third quadrant indicates that the area itself and the surrounding area at a lower 
level(Low-Low); the fourth quadrant indicates that the area itself is high and the surrounding at a lower level(High-Low). The local indicator LISA can study the degree of spatial association, the four quadrants of the agglomeration map have the same meaning as above, the formula is as follows:

$$
\text { Lias }=Z_{i} \sum_{j} W_{i j} Z_{j}
$$

where $Z_{i}$ and $Z_{j}$ are the normalization of observations on regions $\mathrm{i}$ and $\mathrm{j}$, where $\mathrm{W}$ is the spatial weight matrix.

\subsection{Spatial exploratory analysis of China's grain intake}

\subsubsection{Global autocorrelation analysis}

The global Moran index of the daily food intake per capita, the daily rice intake per capita, the daily flour intake per capita, the daily potato intake per capita, the daily dried beans and their products intake per capita is shown in Table 1.

Table 1 Global Moran Index

\begin{tabular}{|c|c|c|}
\hline Index & Moran index & $\mathrm{p}$ value \\
\hline Daily grain intake per capita & 0.363 & 0.000415 \\
\hline Daily rice intake per capita & 0.591 & 0.000000 \\
\hline Daily flour intake per capita & 0.666 & 0.000000 \\
\hline Daily potato intake per capita & 0.627 & 0.000000 \\
\hline Daily dried beans and products intake per capita & 0.301 & 0.002383 \\
\hline
\end{tabular}

As shown in Table 1, the Moran index of China's daily grain intake per capita, daily rice intake per capita, daily flour intake per capita, daily potato intake per capita and daily dried beans and products intake per capita are all positive, indicating that daily grain intake per capita is not random, but presents a positive spatial correlation, with spatial dependence and spatial aggregation. Therefore, the provinces, municipalities directly under the central government and autonomous regions with high (low) grain intake tend to converge with the provinces with high (low) grain intake level, which belongs to the type of high-high (low-low) food concentration. The adjacent regions influence each other and produce dependence.

\subsubsection{Local autocorrelation analysis}

There are obvious regional differentiation and local spatial aggregation in the grain intake of provinces and cities in China.
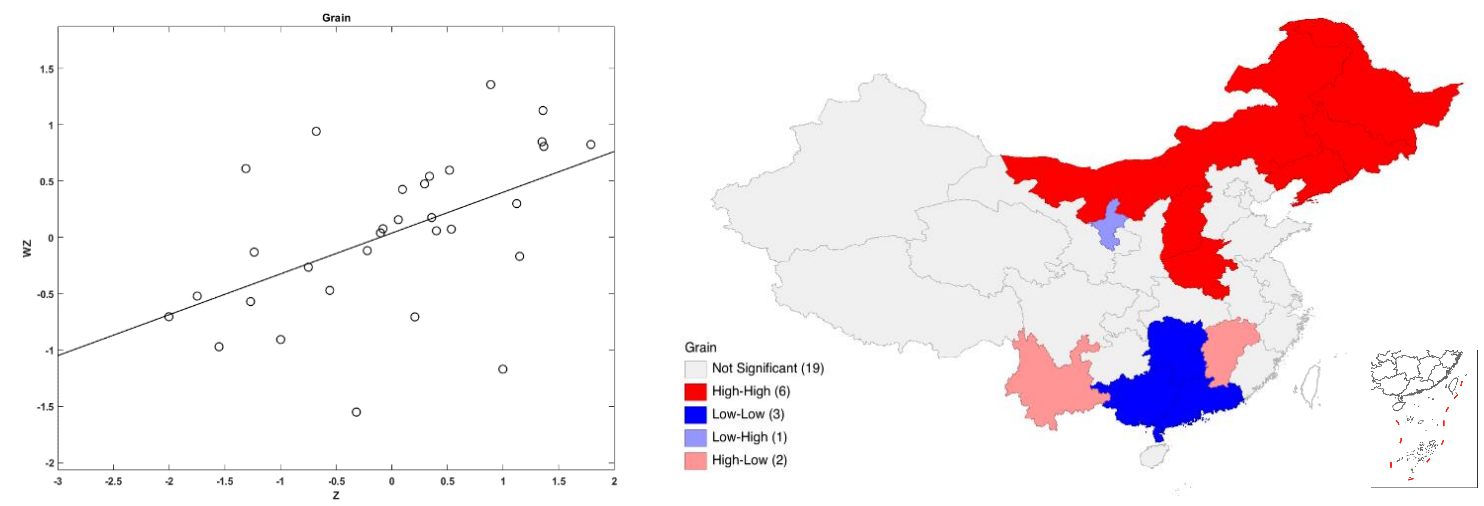

(a) Grain 

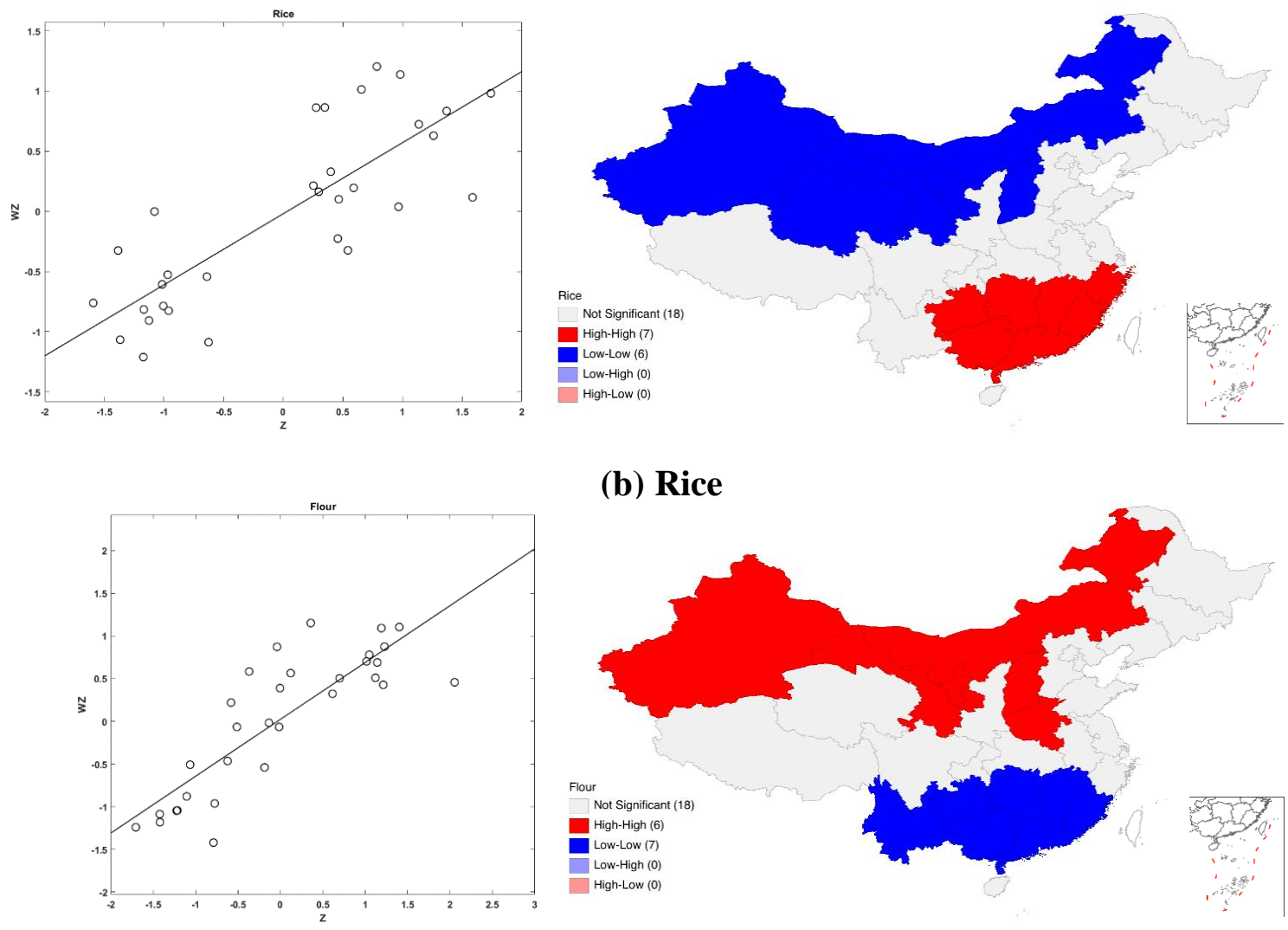

\section{(b) Rice}

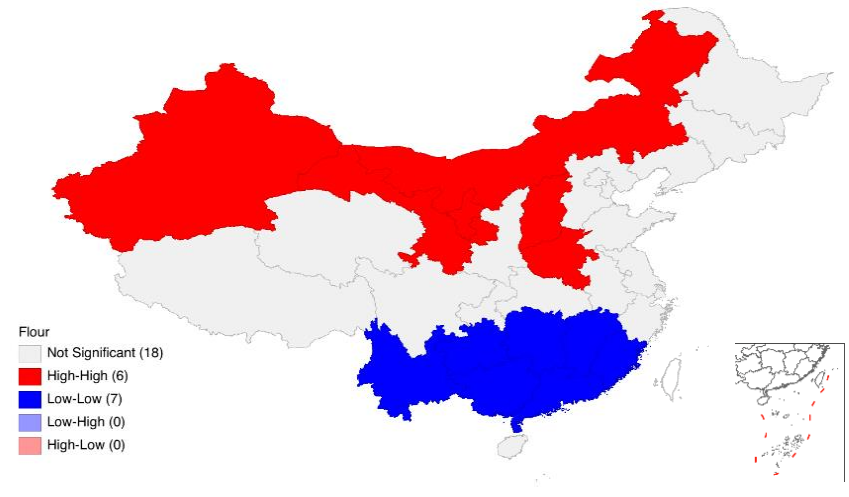

(c) Flour

Fig. 1 Moran scatter plot and Lisa aggregate map of daily grain intake per capita in China

As shown in Fig. 1, the daily grain intake per capita presents a local high-high aggregation mode in the north, a local low-high aggregation mode in Ningxia Hui Autonomous Region, a local low-low aggregation mode in Hunan Province, Guangdong Province, Guangxi Zhuang Autonomous Region in the south, a local high-low aggregation mode in Yunnan Province, Jiangxi Province, and the daily rice intake per capita in the north Xinjiang Uygur Autonomous Region, Qinghai Province, Gansu Province, Shanxi Province, Inner Mongolia Autonomous Region and Ningxia Hui Autonomous Region show a local low-low aggregation mode, while Guizhou Province, Hunan Province, Guangxi Zhuang Autonomous Region, Guangdong Province, Fujian Province and Zhejiang Province in the South show a local high-high aggregation mode; the daily flour intake per capita in Xinjiang Uygur Autonomous Region, Gansu Province in the north Henan Province, Shanxi Province and Inner Mongolia Autonomous Region present the local high-high aggregation mode, and Guizhou Province, Hunan Province, Guangxi Zhuang Autonomous Region, Guangdong Province, Fujian Province and Yunnan Province in the South present the local low-low aggregation mode, which is contrary to the spatial aggregation of the daily rice intake per capita.

\section{Hierarchical Clustering Analysis of China's Grain Intake}

To further analyze the differences and similarities in grain diets in Chinese provinces, we use the four grains per capita daily intake of various provinces and cities in China to conduct spatial cluster analysis of various provinces and cities. 


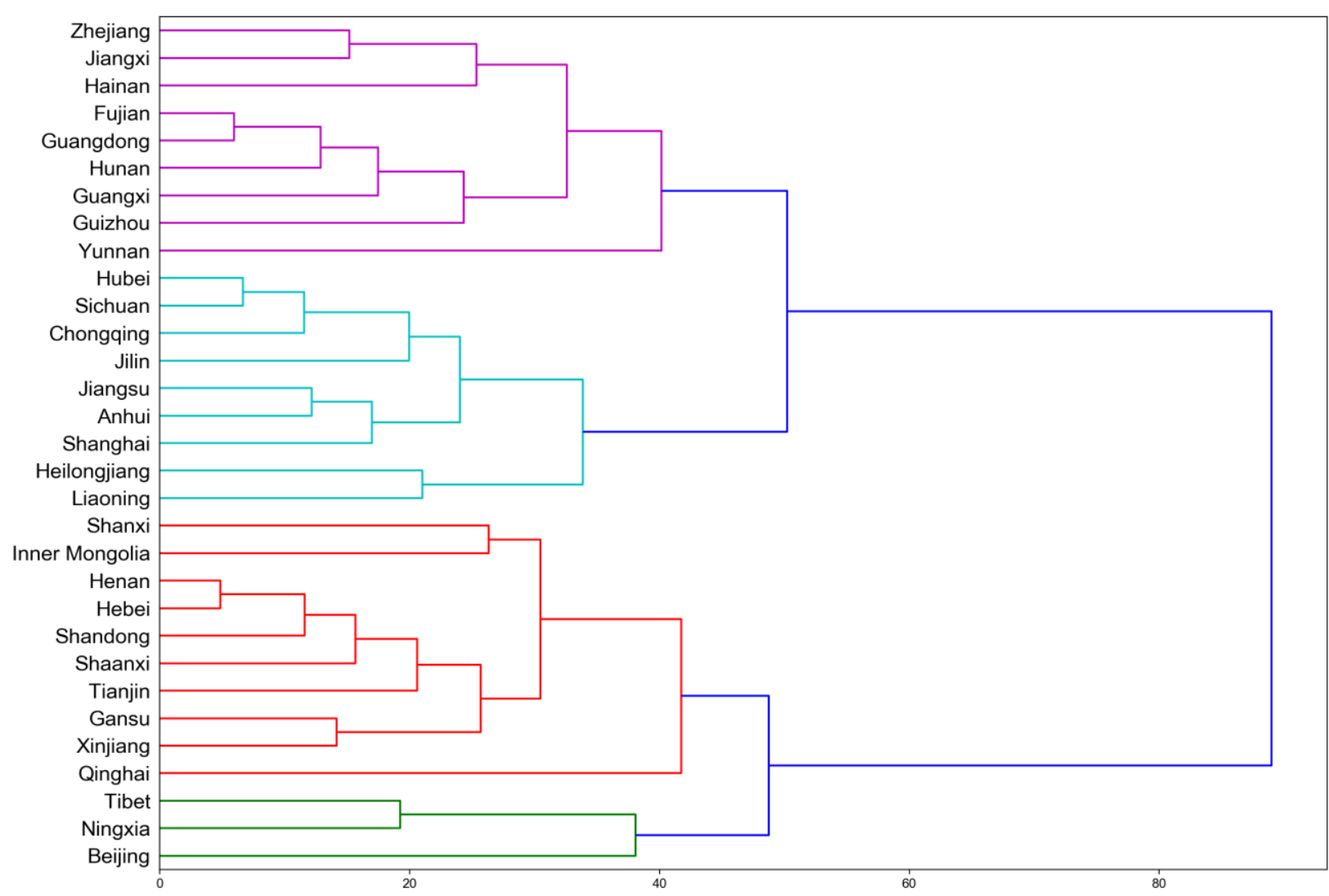

Fig. 2 Schematic diagram of clustering results

As shown in Fig. 2, spatial clustering analysis is carried out according to the daily intake of four grain per capita in each province and city, which are mainly divided into four categories. The first category is Hunan Province in Central China, Zhejiang Province, Jiangxi Province and Fujian Province in East China, and Guangdong Province and Hainan Province in South China, and Guangxi Zhuang Autonomous Region, Yunnan Province and Guizhou Province in the southwestern region, this type of province and city is mainly based on rice intake, geographically located in southern China. The second category is Hubei Province in Central China, Jiangsu Province, Anhui Province and Shanghai in East China, Sichuan Province and Chongqing City in Southwest China, Heilongjiang Province, Jilin Province and Liaoning Province in Northeast China, the provinces and cities in this category mainly focus on rice and flour, the provinces and cities in this category mainly in the northeast, east and southwest regions of China. The third category is Shaanxi Province, Gansu Province, Qinghai Province and Xinjiang Uygur Autonomous Region in Northwest China, Hebei Province, Inner Mongolia Autonomous Region, Shanxi Province and Tianjin City in North China, Shandong Province in East China, and Henan Province in Central China. The provinces and cities mainly focus on flour, and the geographical location is mainly located in the northwestern region of China and the north of China. The fourth category is the Tibet Autonomous Region, Ningxia Hui Autonomous Region, and Beijing, this type of province and city is mainly based on rice and noodles, and the daily grain intake per capita is small.

\section{Conclusion}

In this paper, we explored the spatial correlation and differences in grain intake of Chinese residents. China's grain intake has a positive correlation in geography. Flour and potato intake has a local positive correlation in northern provinces and cities, rice intake has a local positive correlation in southern provinces and cities, and dried beans and its products has local positive correlations throughout the country. Spatial clustering analysis is carried out according to the daily intake of four grain per capita in each province and city, which are mainly divided into four categories. The results 
of this paper have reference value for the formulation of national grain reserve strategy and trade strategy.

\section{Acknowledgment}

This project is supported by the Key Project of Chinese Academy of Sciences (No. KJZD-EW-G20), Projects of Social Science Program of Beijing Education Commission (No. SM201810037001 and SM201910037004) and Beijing Social Science Foundation (No. 18GLB022).

\section{References}

[1] International Food Policy Research Institute, Global Hunger Index, Washington, 2016.

[2] Cheng Y Q, Zhang P Y. Regional Patterns Changes of Chinese Grain Production and Response of Commodity Grain Base in Northeast China, SCIENTIA GEOGRAPHICA SINICA, 5 (2005) 310.

[3] Gao F. Regional changes in China's grain production: 1978-2003, MANAGEMENT WORLD, 9 (2005) 70-78, 87.

[4] Zheng Y G, Kuang C J, Jiao H P. Discussion on the Causes of the Evolution of the South Grain Northward Transfer to the Northern Grain South Movement--Comparing the Advantages and Consumption Comparison of Grain Production Development in the Two Regions of South and North China, Chinese economic history Research, 1 (1999) 99-106, 162.

[5] Qu R X, Liu Y S. The regional pattern of China's grain production since the 1990s, China Agricultural Resources and Regional Planning, 29 (2008) 49-54.

[6] Zhang H, Wang D L, Qu B X, et al. Research on the comparative advantage of major grain varieties in China. China Agricultural Resources and Regional Planning, 25 (2004) 16-20.

[7] Wang X C, Xu F. Regional change characteristics and policy recommendations for grain production in China. Finance and Economics, 4 (2012) 80-88.

[8] Zhou Q F, Dai E F, Wu S H, et al. China's food supply and demand balance risk research under future climate change scenarios. Journal of Peking University (Natural Science Edition), 47 (2011) 1105-1115.

[9] Fang Y P, Tang Y, Hu J. Spatiotemporal evolution of food security pattern and its influencing factors under the background of urbanization-Based on the spatial econometric analysis of China's provinces, Lingnan Academic Journal, 5 (2016) 114-124.

[10] Suresh B, Ergeneman A. A framework for evaluating food security and nutrition monitoring systems. African Journal of Food Agriculture and Nutritional Development (AJFAND), 2005.

[11] Lu W C, Mei Y, Li Y L. Regional changes in China's grain production: the effects of humanland relations, non-agricultural employment and labor compensation, China Population Science, 3 (2008) 20-28,95.

[12] Pan J H, Zhang J H, Hu Y X. The spatial and temporal pattern of per capita grain occupation and its driving force in the county of Gansu Province in the past 20 years, Journal of Natural Resources, 31 (2016) 124-134. 